$1.0(0-20) \mathrm{p}<0.0001$; Casual partners: pre-pandemic: $1.0(0-$ 75); during-pandemic1: $0.0 \quad(0-25) \quad \mathrm{p}<0.0001$, during-pandemic2: $1.0(0-20), p=0.004)$. Among those tested both preand during-pandemic2, $(\mathrm{n}=96)$ STI prevalence was similar (gonorrhea: $14.1 \%$ vs. $15.8 \%, \mathrm{p}=0.847$; chlamydia: $7.0 \%$ vs. 9.5\%, p=0.527).

Conclusions Although overall and casual sex partnerships declined significantly during- compared to pre-pandemic, STI prevalence was similar. The observed decreased sex partnerships may not have substantially altered transmission dynamics or were offset by increased prevalence due to limited access to testing/treatment. Improved understanding of how COVID19 mitigation measures alter STI transmission dynamics is needed.

\section{P451 FACTORS ASSOCIATED WITH GROUP SEX IN HETEROSEXUAL MALES AND FEMALES ATTENDING A SEXUAL HEALTH CLINIC IN MELBOURNE, AUSTRALIA}

\footnotetext{
${ }^{1,2} \mathrm{H}$ Constantinou* ${ }^{*}{ }^{1,2} \mathrm{C}$ Fairley, ${ }^{1,2} \mathrm{C}$ Bradshaw, ${ }^{3} \mathrm{E}$ Choi, ${ }^{1} \mathrm{~K}$ Maddaford, ${ }^{1,2} \mathrm{~T}$ Phillips, $1,2,{ }^{3} \mathrm{E}$ Chow. 'Melbourne Sexual Health Centre, Alfred Health, Melbourne, Australia; ${ }^{2}$ Central Clinical School, Monash University, Melbourne, Australia; ${ }^{3}$ School of Nursing, The University of Hong Kong, Hong Kong
}

10.1136/sextrans-2021-sti.463

Background Group sex participation carries a higher risk of sexually transmitted infection (STI) transmission. Research on group sex has predominately analysed men who have sex with men, while there have been a small number on heterosexuals. This study aimed to explore the factors associated with group sex among heterosexuals.

Methods A cross-sectional survey was conducted amongst heterosexuals aged $\geq 16$ years attending the Melbourne Sexual Health Centre between March and April 2019. Participants completed a survey about participation in group sex, methods used to meet partners, casual and/or regular partners, and intravenous drug use (IVDU) in the previous 3 months. HIV and STI (chlamydia, gonorrhoea, syphilis) diagnoses were extracted. A multivariable logistic regression was conducted to identify the factors associated with group sex participation.

Results A total of 698 participants (325 males, 373 females) were included and $4.7 \%$ (33/698) had participated in group sex in the previous 3 months. There was no difference between the proportion of males $(6.5 \%, 21 / 325)$ and females $(3.2 \%, 12 / 373)$ participating in group sex $(p=0.198)$. The proportion who participated in group sex increased with age $(2.1 \%$ in $16-24$ years, $5.5 \%$ in $25-34$ years, $7.8 \%$ in $\geq 35$ years, ptrend $=0.010$ ). Meeting partners at sex-venues (e.g. brothel) was associated with the highest odds of participating in group sex $(\mathrm{AOR}=5.74,95 \% \mathrm{CI}: 1.20-27.44)$, followed by dating-apps $\quad(\mathrm{AOR}=2.99, \quad 95 \% \mathrm{CI}: 1.36-6.58), \quad$ friends/family $(\mathrm{AOR}=2.99$, 95\%CI:1.34-6.69) and social-venues (e.g. bar) $(\mathrm{AOR}=2.73$, 95\%CI:1.18-6.30). Group sex was strongly associated with STI positivity (AOR=6.24, 95\%CI:2.41-16.13). There was no association between group sex and HIV positivity, casual and/or regular partners, or IVDU.

Conclusion Heterosexuals participating in group sex had a sixfold risk of testing positive for STIs and were most likely to meet sexual partners at sex-venues. Including group sex in a sexual history is useful to determine STI risk. Safe sex messages on group sex at sex-venues would be beneficial.

\section{P452 COVID UNDER THE SHEETS, A STI CLINIC SURVIVES LOCKDOWN!}

${ }^{1} \mathrm{P}$ Sharma*, ${ }^{1} \mathrm{P}$ Pathak, ${ }^{1,2} \mathrm{~S}$ Salgaonkar, ${ }^{1} \mathrm{D}$ Rowley*. 'Midland Regional Hospital Portlaoise, Portlaoise, Ireland; ${ }^{2}$ Medical Univeristy of Warsaw, Warsaw, Poland

\subsection{6/sextrans-2021-sti.464}

Background Our aim principally was to assess our readiness to remain open as we provide an essential public health service namely the treatment of infectious diseases. Facing this pandemic with limited preparation time, the journey of our sexual health clinic had to evolve multiple times in order in to provide optimal care for our patient population.

Method A retrospective study was conducted of all consultations during March - June 2020, which was Ireland's first lockdown period and was compared to the same period in 2019.

Results 251 symptomatic people attended the clinic in 2019 compared to 129 attendees in 2020. We assume; this was due to our national lockdown due to a global pandemic. Of note, a doubling of Herpes Simplex Virus (HSV) rates was noted from $4 \%$ in 2019 to $8 \%$ in 2020 . Non- sexually transmitted infections increased from $11 \%$ in 2019 to $53 \%$ in 2020, These included genital dermatoses, non-specific urethritis, and testicular pathologies such as epididymitis.

Conclusion Our sexual health clinic adapted overnight to the challenges of Covid-19 with virtual consults being one of many new techniques that were employed. The high number of symptomatic patients certainly surprised us but also helped us in understanding human nature and their craving for social interaction. 